\title{
Features of Blastocystis spp. in xenic culture revealed by deconvolutional microscopy
}

\author{
Robyn Nagel $^{1}$ - Christian Gray ${ }^{2} \cdot$ Helle Bielefeldt-Ohmann ${ }^{1,3} \cdot$ Rebecca J. Traub ${ }^{4}$
}

Received: 1 May 2015 / Accepted: 14 May 2015 / Published online: 22 May 2015

(C) The Author(s) 2015. This article is published with open access at Springerlink.com

\begin{abstract}
Blastocystis spp. are common human enteric parasites with complex morphology and have been reported to cause irritable bowel syndrome (IBS). Deconvolutional microscopy with time-lapse imaging and fluorescent spectroscopy of xenic cultures of Blastocystis spp. from stool samples of IBS patients and from asymptomatic, healthy pigs allowed observations of living organisms in their natural microbial environment. Blastocystis organisms of the vacuolated, granular, amoebic and cystic forms were observed to autofluorescence in the 557/576 emission spectra. Autofluorescence could be distinguished from fluorescein-conjugated Blastocystis-specific antibody labelling in vacuolated and granular forms. This antibody labelled Blastocystis subtypes 1,3 and 4 but not 5 . Surface pores of $1 \mu \mathrm{m}$ in diameter were observed cyclically opening and closing over $24 \mathrm{~h}$. Vacuolated forms extruded a viscous material from a single surface point with coincident deflation that may demonstrate osmoregulation. Tear-shaped granules were observed exiting from the
\end{abstract}

Electronic supplementary material The online version of this article (doi:10.1007/s00436-015-4540-x) contains supplementary material, which is available to authorized users.

Robyn Nagel

robyn@tgclinic.com.au

1 School of Veterinary Science, University of Queensland, Gatton, Queensland, Australia

2 Plymouth University Peninsula Schools of Medicine and Dentistry, Plymouth University, Plymouth, UK

3 Australian Infectious Diseases Research Centre, University of Queensland, St Lucia, Queensland, Australia

4 Faculty of Veterinary and Agricultural Sciences, University of Melbourne, Parkville, Victoria, Australia surface of an amoebic form, but their origin and identity remain unknown.

Keywords Blastocystis · Deconvolutional microscopy · Autofluorescence

\section{Introduction}

Blastocystis spp. are common enteric, unicellular parasites found in almost every species of animal (Tan 2008). Seventeen different subtypes (STs) defined by the 18SSU of the ribosomal RNA gene are recognised, and ST3 is almost universally the most common of the nine STs found in humans (Stensvold 2012). Blastocystis infection has been linked to irritable bowel syndrome-like symptoms in humans, but epidemiological studies are inconclusive (Scanlan 2012). Many healthy people carry Blastocystis sp., and it remains unclear if the parasite is pathogenic. Pathogenicity has been speculated to relate to parasite subtypes (Eroglu and Koltas 2010; Tan et al. 2006) as well as to the host's immune response (OlivoDiaz et al. 2012).

Analysis of the genome of two different STs of Blastocystis sp. has allowed prediction of genes and proteins (Denoeud et al. 2011) that may be associated with the organism's pathogenic potential. Nevertheless, a lack of knowledge of the basic life cycle and metabolic function of the parasite remains a major limitation to exploiting the use of animal models and in vitro systems to further explore the clinical significance and therapeutic options for the control of this organism.

Studies utilising light microscopy, transmission (TEM), scanning (SEM) and freeze-etch (FE-EM) electron microscopy have described multiple forms of the Blastocystis organism, including vacuolated (VF), granular (GF), amoebic (AF) 
and cystic (CF) forms (Fig. 1). The relationship of these different forms to each other is unclear (Tan 2008), although it is certain that the robust cystic form transmits infection (Moe et al. 1997). Microscopic images have often been obtained from attenuated or axenic cultures. These elegant studies have been useful in describing the intricate ultrastructure and surface morphology of the various forms of Blastocystis spp., but their limitation is that they capture still images of a dead organism separated from the usual microbial environment. In this study, we employed deconvolutional microscopy of xenic cultures of living Blastocystis sp. to obtain time-lapse and threedimensional images of the Blastocystis organisms. This microscope also has the capability to record fluorescence in various light spectra facilitating utilisation of Blastocystis-specific fluorescent antibodies and fluorescent double-stranded deoxyribonucleic acid (DNA) stain,
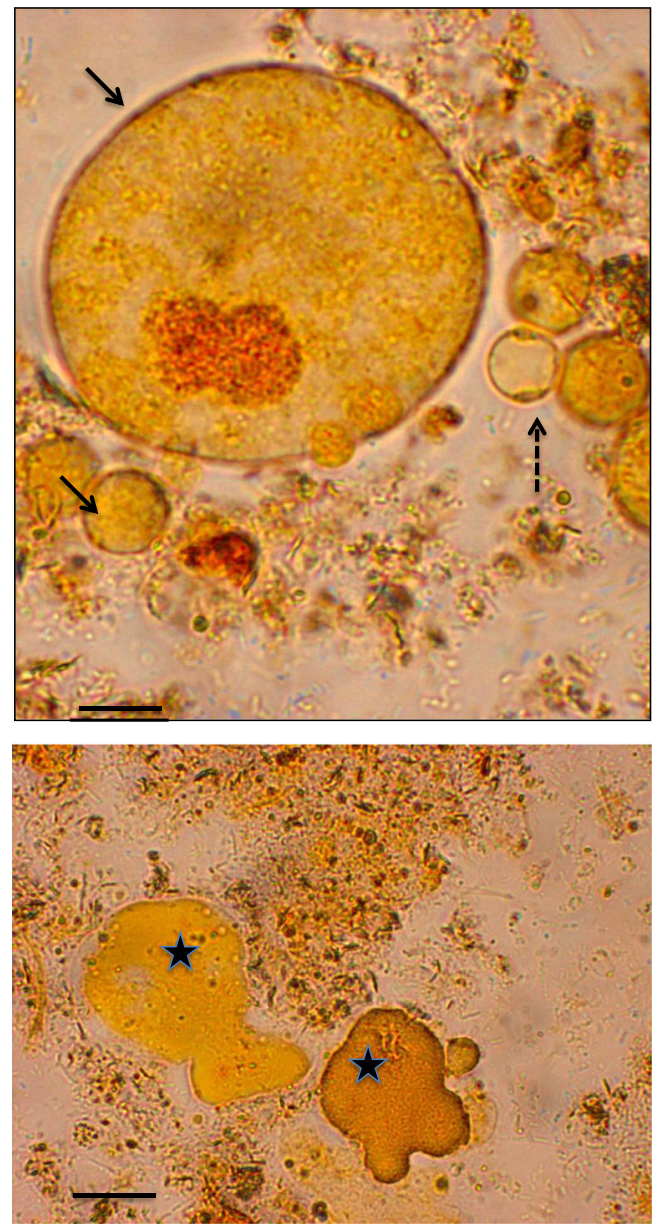

Bar 10um

solid arrow:granular forms

dashed arrow:vacuolated form

star: amoebic forms

Fig. 1 Morphological forms of Blastocystis sp. in xenic culture stained with acridine orange 4',6-diamidino-2-phenylindole (DAPI) (Sigma-Aldrich, Australia).

\section{Materials and methods}

\section{Sample preparation}

Fresh faecal specimens were obtained from irritable bowel syndrome patients positive for Blastocystis carriage and from pigs at the University of Queensland (UQ) Gatton Campus piggery, obtained in accordance with University of Queensland Medical and Animal Ethics Committee approvals numbers 2011000454 and 2012000069 , respectively. Ten grams of faeces was subcultured aerobically for $24-48 \mathrm{~h}$ in Jones medium (Jones 1946) supplemented with $10 \%$ heat-inactivated horse serum. The sediment at the interface between the basal residue in the test tube and the liquid medium was removed for microscopic analysis.

\section{Slide preparation}

Fifteen microliters of fresh sediment was mixed gently with $10 \mu \mathrm{L}$ phosphate-buffered saline and placed on a glass slide. The edges of the slide cover were sealed to prevent air entry to the specimen. An inverted slide was placed into a Deltavision Elite deconvolution microscope (Applied Precision, GE Healthcare, Berthold Australia, Bundoora, Victoria, Australia) and examined with polarised light and with fluorescent filters. Four different filters that allowed excitation and emission ranges from 350 to $700 \mathrm{~nm}$, including DAPI, fluorescein isothiocyanate (FITC), tetramethylrhodamine (TRITC) and cyanine 5 (Cy5) spectroscopy ranges were used for examination. The images were magnified 20 times or 40 60 times with oil immersion and repeated images were taken at cross sections through the specimen or at different time intervals. Time-lapse microscopy was performed over $24 \mathrm{~h}$ with images taken every $15 \mathrm{~min}$. The environmental chamber was maintained at $37{ }^{\circ} \mathrm{C}$ throughout the microscopy session.

\section{Antibody staining}

FITC-conjugated Blastocystis-specific polyclonal antibody raised against axenic ST3 (Blastofluor ${ }^{\circledR}$; Antibodies Inc., David, CA, USA) was also used to assess the specimens. Two hundred microliters of sediment was incubated with $5 \mu \mathrm{L}$ of Blastofluor ${ }^{\circledR}$ for $30 \mathrm{~min}$ at $37^{\circ} \mathrm{C}$. Staining of double-stranded DNA with DAPI was performed by adding $2 \mu \mathrm{L}$ of stock solution of DAPI $(0.5 \mu \mathrm{g}$ DAPI in $1 \mathrm{~mL}$ PBS $)$ to the prepared microscopy slide and gently mixed before incubating in the dark for $10 \mathrm{~min}$. 


\section{Results and discussion}

\section{Auto- and immunofluorescence}

Autofluorescence was observed in VF, GF and AF and the CF (Fig. 2) of Blastocystis cells. The autofluorescence was greatest in the TRITC excitation/emission light spectra (557/ 576-nM) and appeared to be brightest in the central vacuole, with spots of light emission observed at the surface of the organism. The autofluorescence faded with time and appeared to be optimal in fresh VF forms, CF and least in AF. The autofluorescence was distracting but could be distinguished from the Blastofluor ${ }^{\circledR}$ stain in the VF and GF. The Blastofluor ${ }^{\circledR}$ stained the external cell membrane of VF, GF but not the AF (Fig. 3) and was brightest using the FITC (495/ 515-nm) light spectrum. The Blastofluor ${ }^{\circledR}$ stain could not be reliably differentiated from the strong autofluorescence seen in the CF. The Blastofluor ${ }^{\circledR}$ antibody stained the VF and GF of STs 1, 3 and 4 specimens but did not stain these forms in ST5 pig samples. DAPI staining of VF stained the nuclei on opposite poles of the central vacuole a dense bright blue.

Non-specific green autofluorescence (GAF) was originally thought to be a rare occurrence in microalgae that might prove a useful taxonomic feature. However, over time, GAF has been identified in many different organisms including algae, dinoflagellates, diatoms, cyanobacteria and raphidophytes (Tang and Dobbs 2007). The GAF occurs in the cytoplasm of these microalgae as well as in cysts that have little cytoplasm and is affected by various types of stress on the cell such as light, heat and drying and is caused by molecules other than chlorophyll. The chemical nature of GAF is unknown but is likely caused by multiple compounds or multiple derivatives of a single compound, such as flavin-like molecules, or luciferin compounds (Tang and Dobbs 2007). A variety of stains that fluoresce in the green light spectrum are used to assess morphology and physiological states of organisms and GAF has been reported to interfere with the interpretation of these stains (Tang and Dobbs 2007). This is the first report of autofluorescence by Blastocystis organisms. Blastocystis diverged in evolution at the time of red algae and the genome suggests incorporation of cyanobacteria genes (Denoeud et al. 2011), so the presence of GAF is not unexpected. The GAF was seen strongly in the central vacuole of the VF. Little is known of the chemical constituents of this vacuole except that it stains positively for carbohydrates (Yoshikawa et al. 1995a) and lipids (Yoshikawa et al. 1995b).

The GAF did not interfere with the ability to detect Blastofluor ${ }^{\circledR}$ staining at the surface of the organism in the VF and GFs but could not be differentiated from GAF in cysts. Blastocystis cysts are difficult to identify in stools, but as GAF is a relatively non-specific finding in microalgae, screening with fluorescent filters may not aid detection of the CF. Blastofluor ${ }^{\circledR}$ did not stain the AF of Blastocystis suggesting that the surface antigens of this form differ from the VF and GF. Blastofluor ${ }^{\circledR}$ is a polyclonal antibody raised in rabbits to axenic cultures of ST3 and has been shown to react to Blastocystis organisms with no cross reactivity with Giardia duodenalis, Entamoebae histolytica or Cryptosporidium spp. (Dogruman-Al et al. 2010). Blastocystis immunolabelling with Blastofluor ${ }^{\circledR}$ has previously been shown to stain faecal samples of steers, goats and humans, but not pigs after prolonged (up to a year) storage in formalin (Gould and Boorom 2013). This is consistent with our own observations in which no Blastofluor ${ }^{\circledR}$ staining was detected in our ST5 pig samples. ST5 organisms are commonly detected in pigs worldwide and locally, $100 \%$ pigs have previously been

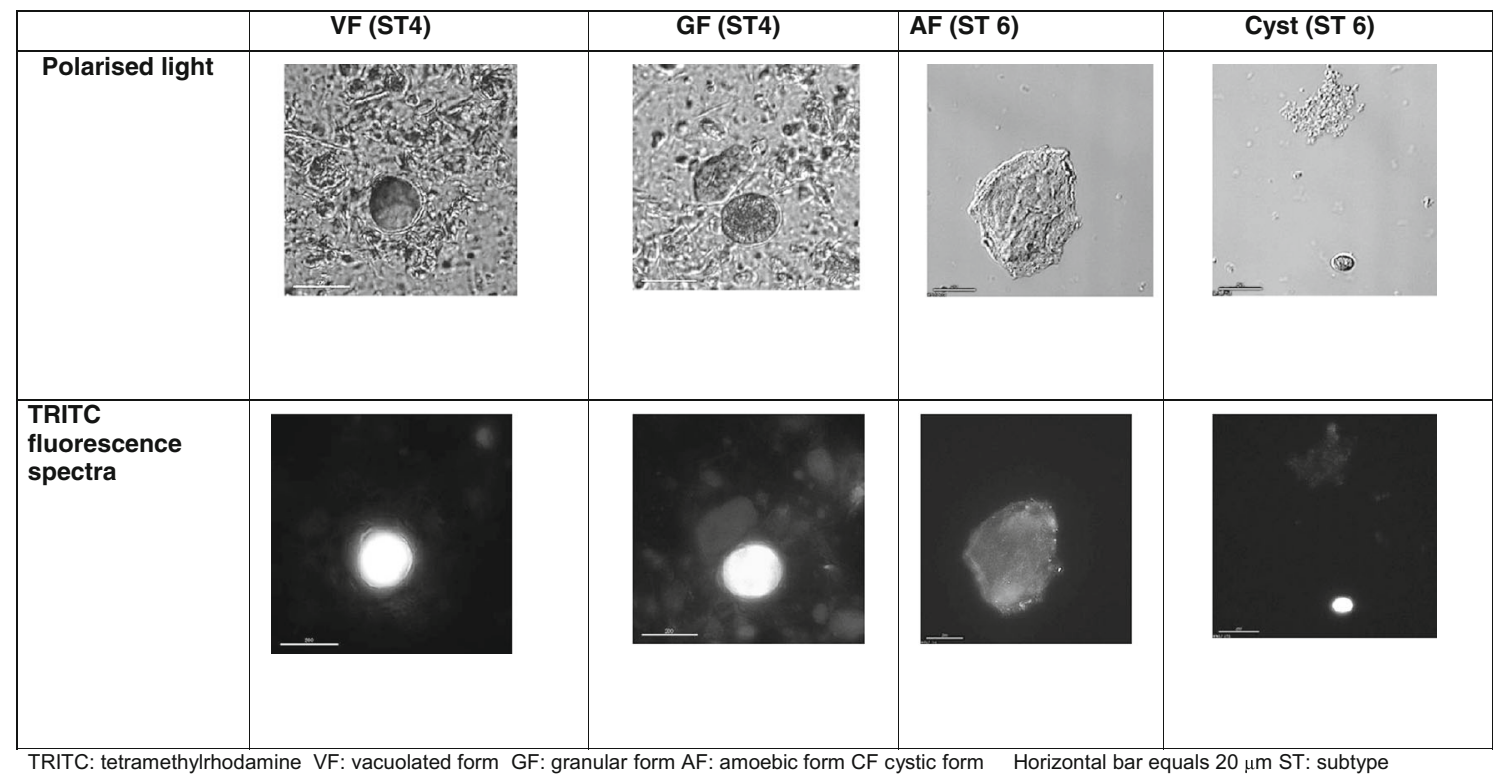

Fig. 2 Autofluorescence of different morphological forms of Blastocystis spp. 


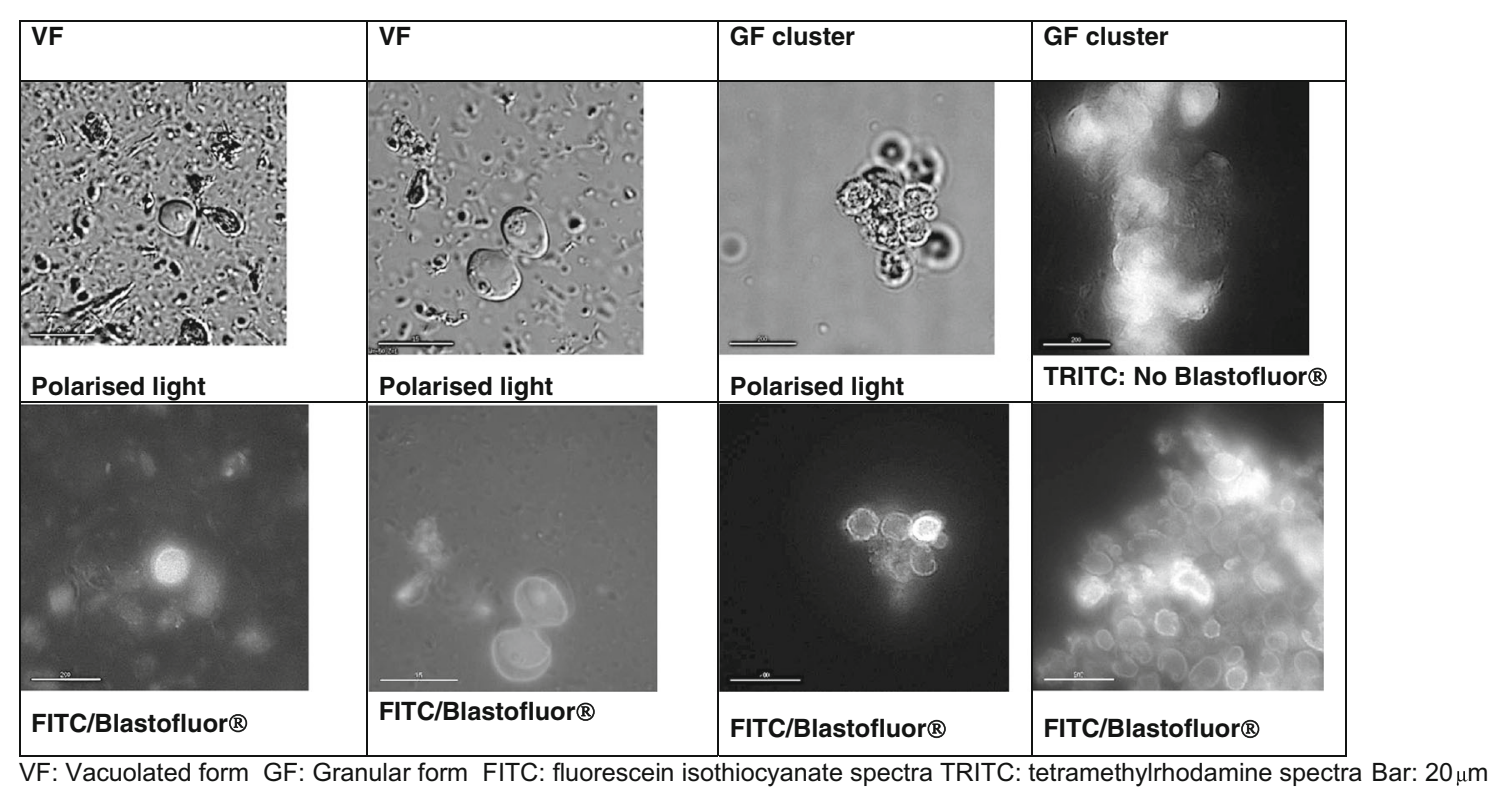

Fig. 3 Different morphological forms of Blastocystis spp. stained with Blastofluor ${ }^{\circledR}$

shown to carry Blastocystis sp. ST5, and $7 \%$ harbour mixed infection with ST1 (Wang et al. 2014). This suggests that the surface antigens display homology between some STs (ST 1, 3,4 ) but that ST5 organisms differ in some respect to some surface antigens.

\section{Cell granules and time-lapse microscopy}

Detailed views of Blastocystis organisms were obtained, but apart from observing binary fission developing in vacuolated and granular forms, we did not observe changes from one morphological form to another. It was difficult to keep the Blastocystis organisms in the field of view despite using the cell tracking facility of the microscope. The Blastocystis cells were fragile and would often either rupture (Fig. 4) or drift away out of the field of view over the $24 \mathrm{~h}$ of recording, preventing observation of progressive cell changes past a certain point (Fig. 5). One GF, observed over $6 \mathrm{~h}$ in total, that appeared stationary on the slide displayed a large central granule moving rapidly within the GF (Fig. 6, Supplementary video file 1). Another time-lapse study of a granular AF showed the form enlarging and losing granularity on the surface with subsequent expulsion of small tear-shaped granules. These granules appeared to arise from the surface of the organism or track along fissures to the surface (Fig. 7). These granules varied in size from 1 to $3 \mu \mathrm{m}$ and appeared to have a central dark spot surrounded by clear cytoplasm with a thick dark surface membrane. The release of the granules from the surface was best appreciated in the video images (Supplementary video file 2). Granules that appear to have tracked along fissures are identified as an area of interest in Fig. 7.
The life cycle of Blastocystis spp. remains elusive and the function of the multiple inclusion granules present in the GF is still unknown. These granules have been proposed to have functions related to metabolism, storage or reproduction (Zierdt 1991). DAPI has been shown to stain VF doublestranded DNA strongly in the nucleus and weakly in the mitochondria-like organelles (Stenzel and Boreham 1996) but did not stain the central vacuole or the granules of GFs (Matsumoto et al. 1987). These granules have been classified into three different types based on TEM studies (Tan and Zierdt 1973), namely myelin-like inclusions, crystalline granules and lipid droplets (Dunn et al. 1989). None of these previous microscopy studies have supported the hypothesis that these central granules constitute a reproductive phase. The cause of the movement of the large central granule seen within the GF in this study (Fig. 6) is not understood, and simple Brownian motion cannot be excluded.

The small granules that were expelled from the granular AF are intriguing (Fig. 7) but DAPI stains were not performed on the AF to confirm a reproductive role. AF are reported to occur commonly in faeces (Stenzel and Boreham 1996), particularly in patients with symptomatic diarrhoea (Tan and Suresh 2006), but only rarely in culture. Asexual binary fission of VF and GF occur commonly in culture but other routes of reproduction have not been proven (Tan and Stenzel 2003). It is possible that the gut microbiota is necessary for completion of the normal life cycle of Blastocystis and that sexual replication may occur only in primed AF in the gut.

\section{Other surface changes observed over time}

Surface pores, with an approximate diameter of $1 \mu \mathrm{m}$, were seen on the external surface of the cell membrane of a partially 
Fig. 4 Vacuolated form of Blastocystis sp., 15-min timelapse sequence
1

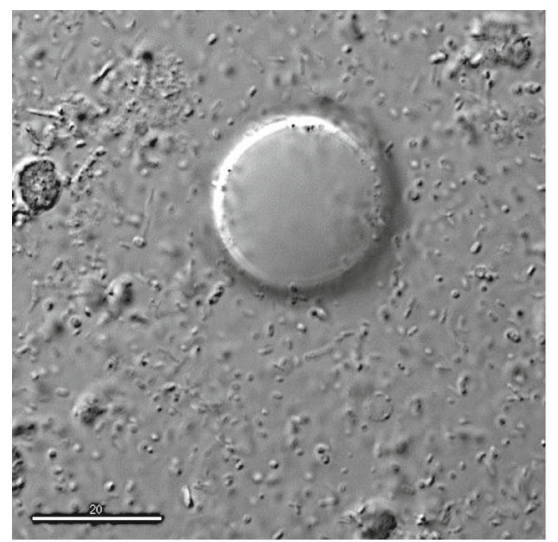

3

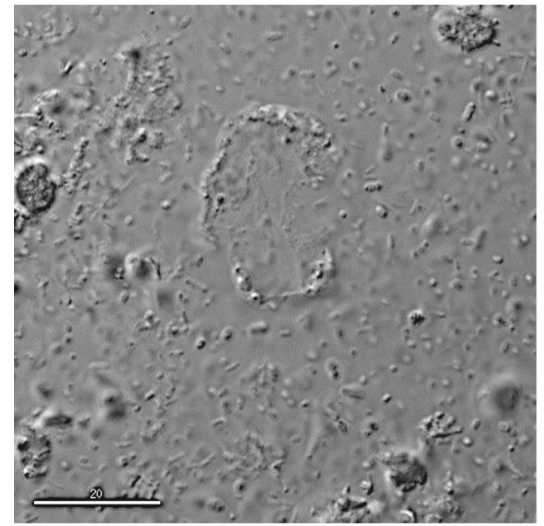

Bar $20 \mu \mathrm{M}$
2

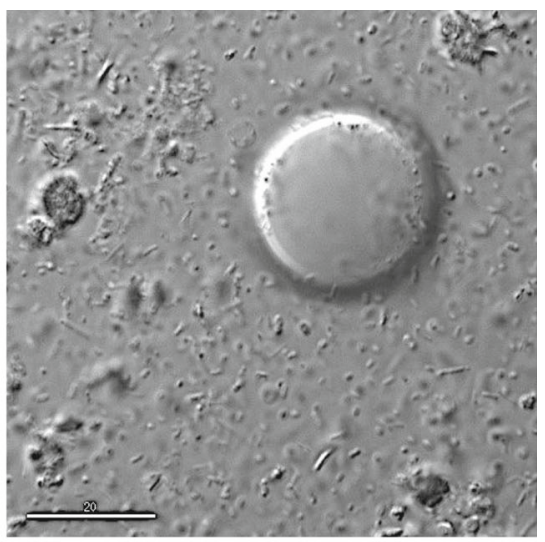

4

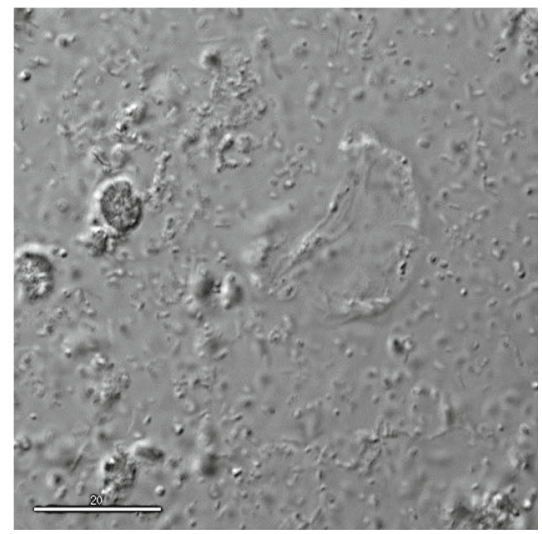

ruptured ST3 Blastocystis organism (either a VF or a GF). The time-lapse recording of this event shows a total of 15 pores opening and closing (Supplementary video file 3). They appear to open and close fully over $1 \mathrm{~h}$ during four 15-min interval observations. The regular movement of the pores continued for the length of the $24 \mathrm{~h}$ study. A time interval sequence is shown with two pores in the foreground demonstrating fully open and closed pore positions in Fig. 8.

The presence of surface pores concurs with previous EM findings. Using TEM, four morphological types of electron dense pits have been described on the surface membrane of the cell wall of Blastocystis sp., namely bars, cup-shaped pits, alveolate pits and tubular pits (Dunn et al. 1989). SEM studies have also confirmed the presence of small indentations on the external cell wall membrane, with fewer indentations noted on cells in prolonged culture (Cassidy et al. 1994). FE-EM has shown pores, approximately $0.5 \mu \mathrm{m}$ in diameter, on the external surface of the outer cytoplasmic membrane that correspond to indentations on the inner surface of the outer cytoplasmic membrane (Tan et al. 1974), suggesting that pores may connect the outer and inner cytoplasmic membrane into the central vacuole. Unfortunately, FE-EM damages the
Fig. 5 Xenic culture of Blastocystis granular forms, timelapse images over $24 \mathrm{~h}$
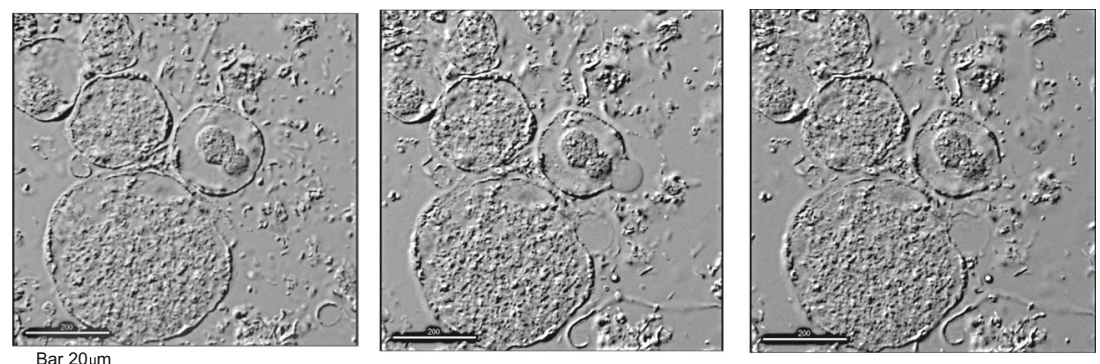


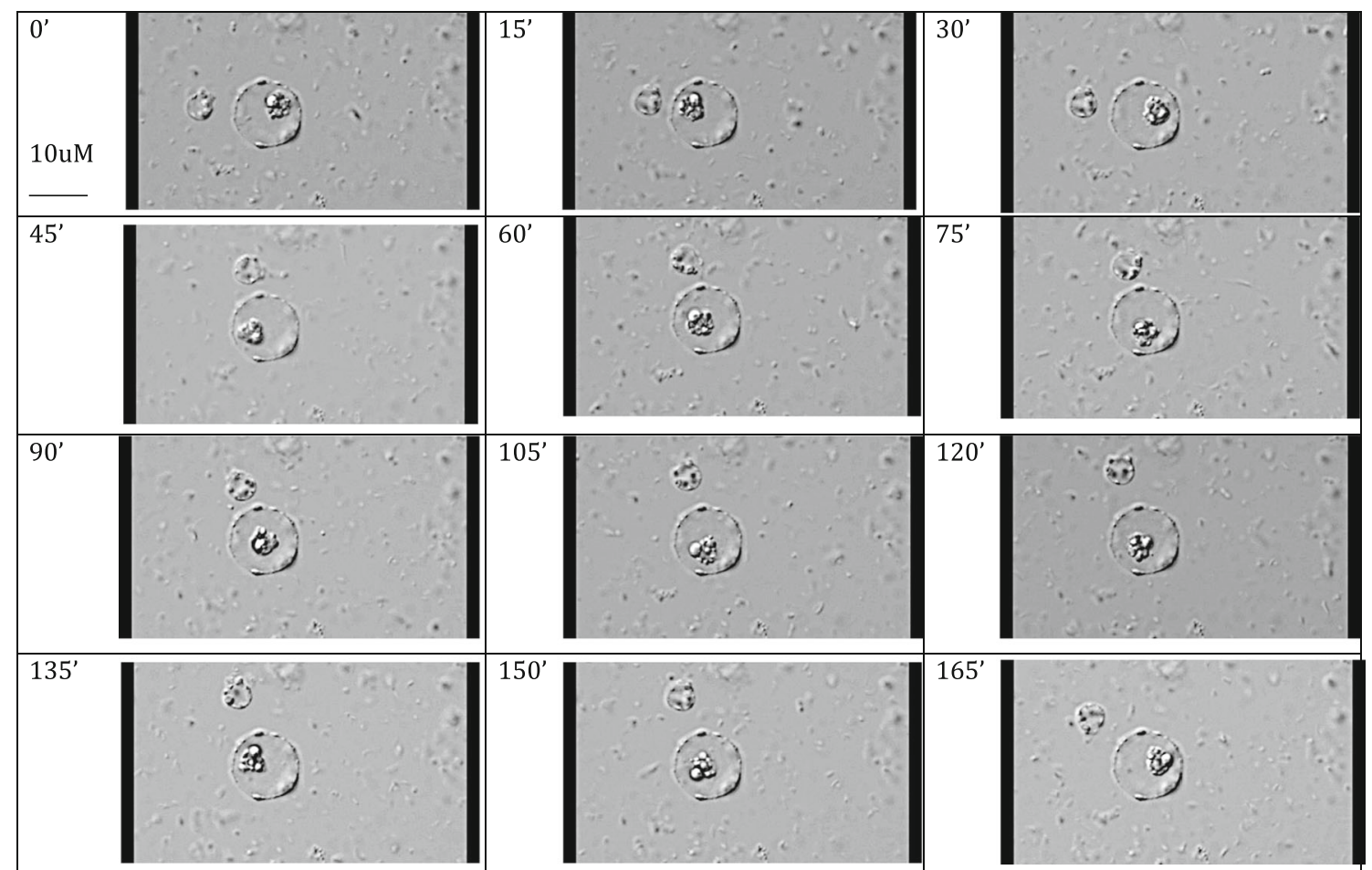

Fig. 6 Blastocystis with moving central granule sequential images over 15-min time intervals

external layer of the outer cytoplasmic membrane so that FEEM cannot confirm if these pores connect all the way through the cell surface.

Another time-lapse series of images showed extrusion of a viscous material apparently from one single point on the external cell membrane of two VFs of Blastocystis (Fig. 9) (Supplementary video file 4). The Blastocystis organisms were first seen to increase in size over $13 \mathrm{~h}$ then deflate, but without disintegrating, as this substance extruded. This extrusion process took $11.5 \mathrm{~h}$ in total, and the extruded material had
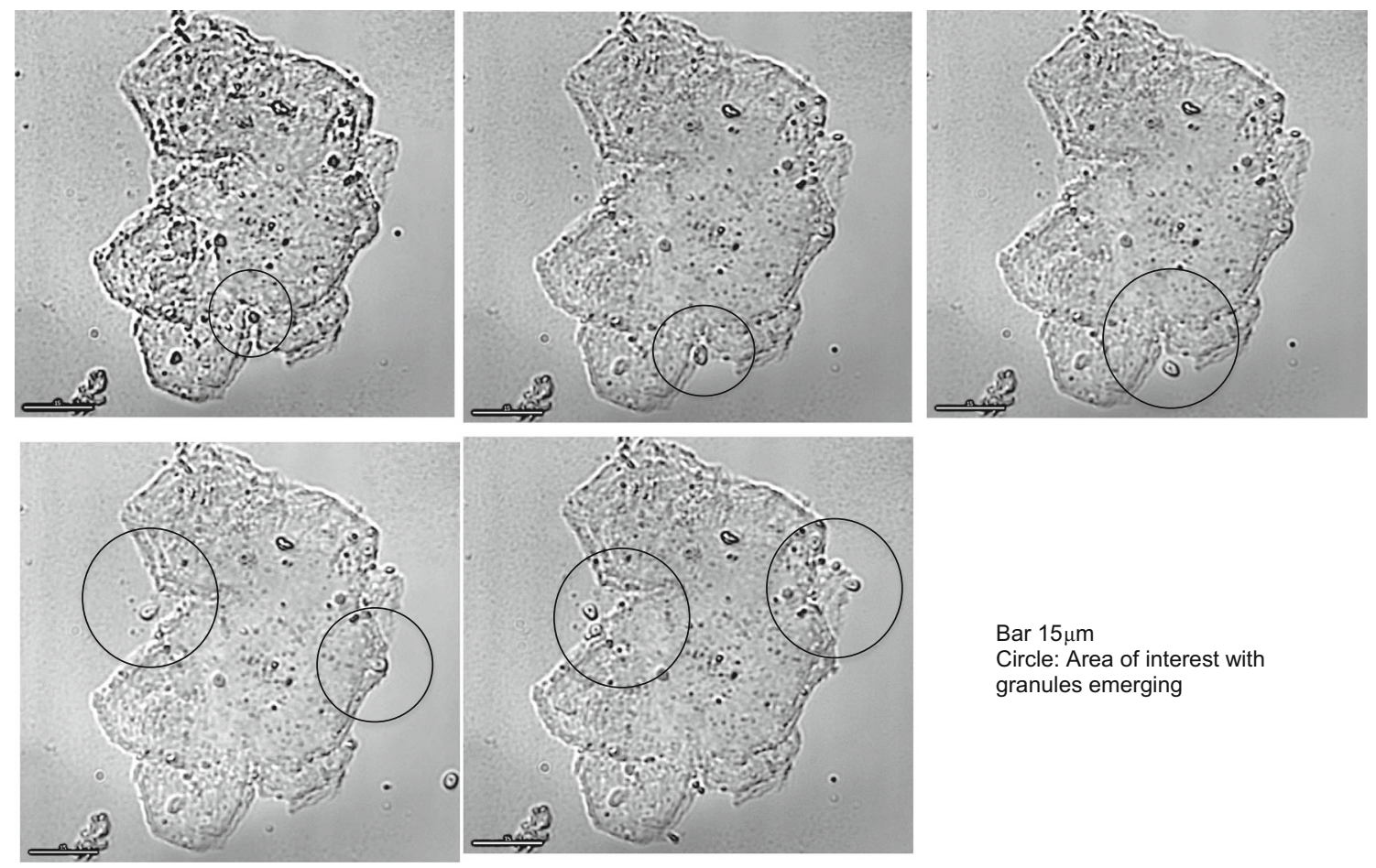

Bar $15 \mu \mathrm{m}$

Circle: Area of interest with

granules emerging

Fig. 7 Granules exiting Blastocystis amoebic form in xenic culture, time-lapse images taken over $24 \mathrm{~h}$ 
Fig. 8 Blastocystis form in xenic culture showing pores on surface
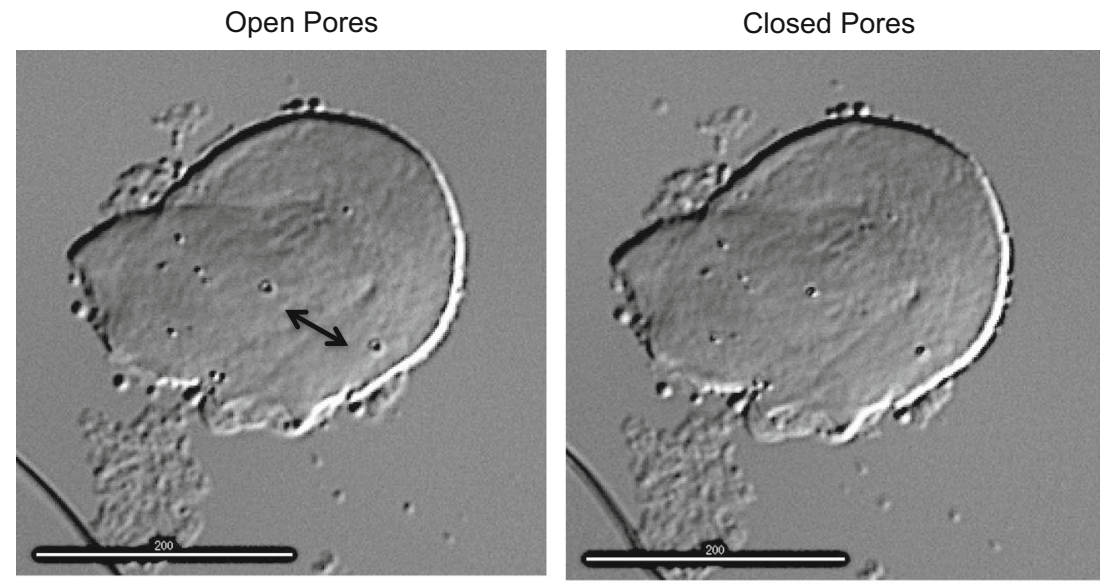

Bar: $20 \mu \mathrm{M}$

$\longleftrightarrow$ marks two pores of interest

an amoebic shape. A separate DAPI-stained slide (with multiple images taken at cross sections through the slide, but not time-lapse) stained nuclei in adjacent VFs bright blue, but there was no discrete nuclear material staining seen in the adjacent amoebic shape. This amoebic-shaped structure appeared very similar in shape to the extruded material observed in the previous slide (Fig. 10). The centre of this amoebic structure stained a light diffuse blue, and the surface is covered in granules that do not fluoresce with the DAPI stain.

This curious extrusion of viscous material from the VF occurring over many hours was observed to occur with deflation but conservation of the VF (Fig. 9). Typically, VFs have only a fine rim of cytoplasm surrounding a large central vacuole. The large amount of this viscous material extruded from
1

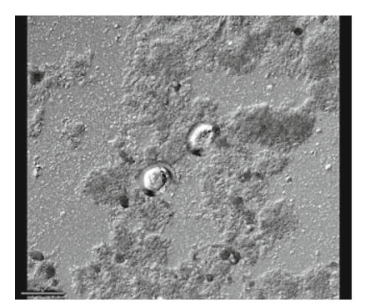

5

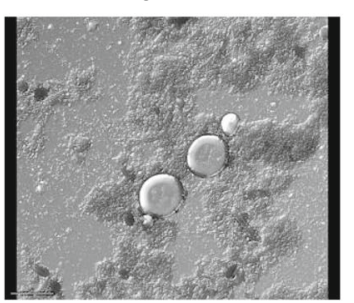

9

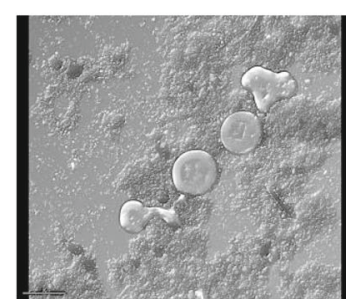

2

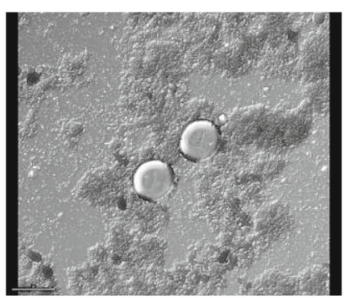

6

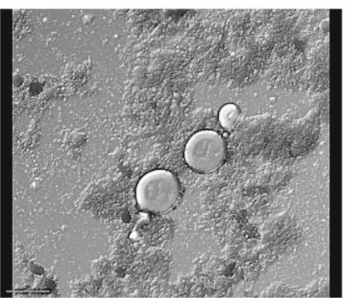

10

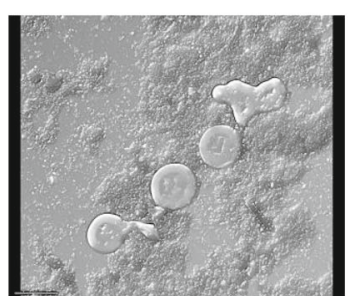

3

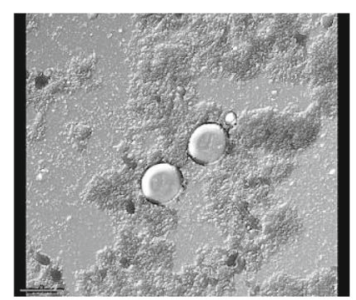

7

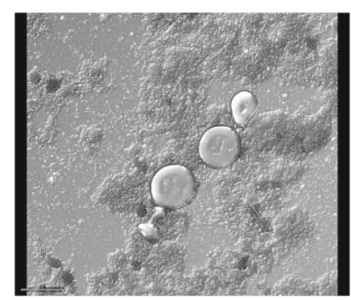

11
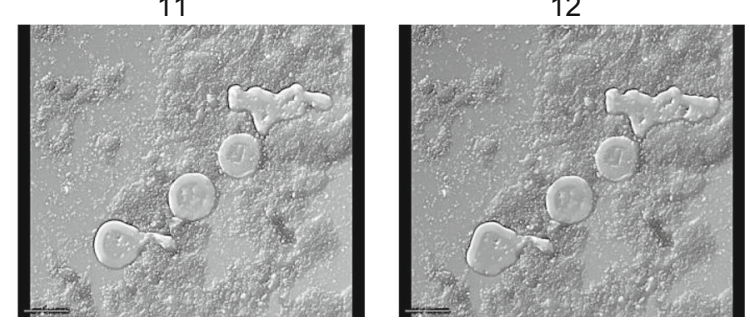

4

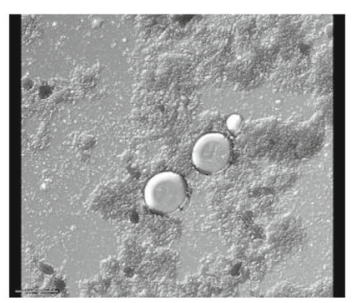

8

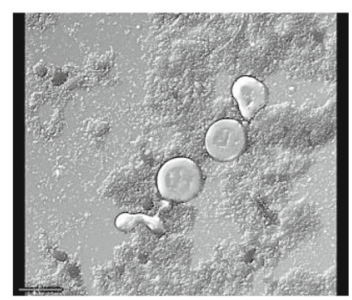

12
Bar: $0.9 \mu \mathrm{M}$

1-2 images: time interval: 13 hours $2-12$ images: 11.5 hours

Fig. 9 Xenic culture of Blastocystis vacuolated form with extrusion, time-lapse images 
Fig. 10 Fluorescent microscopy of Blastocystis forms stained with DAPI

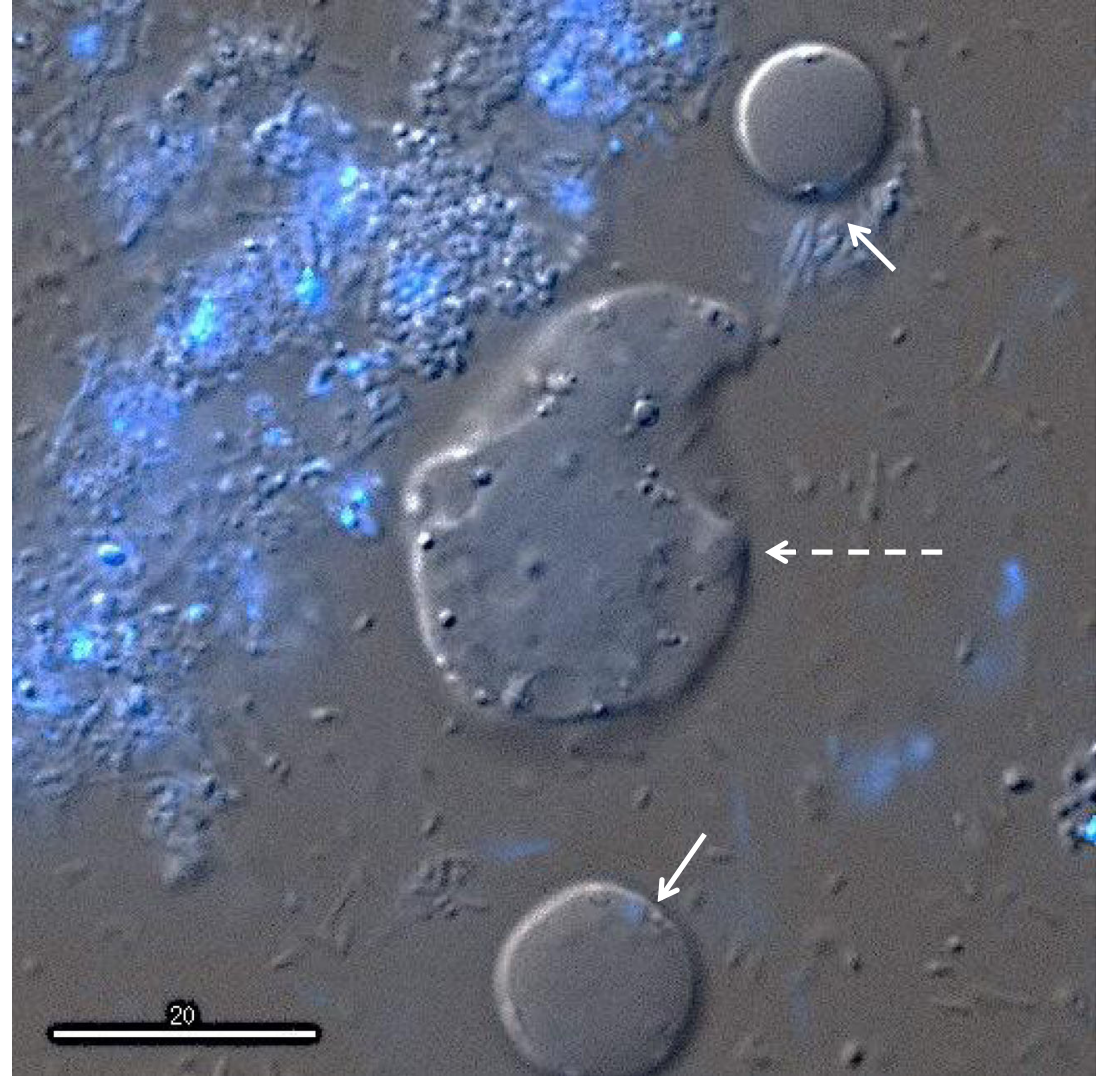

Bar: $20 \mu \mathrm{m}$

Solid arrows: Vacuolated forms of Blastocystis with nuclei at tip of arrow Dashed arrow: Amoebic shape

DAPI: 4',6-diamidino-2-phenylindole a single point occurring simultaneously with deflation of the VF is consistent with a direct connection from the surface to the central vacuole. This fluid extrusion may be occurring through the surface pores, although it was interesting to note that the fluid only appeared to exit from one place on the surface wall and not from multiple pores.

The regular, repetitive movement of pores opening and closing in these time-lapse images suggests that the movement is active and requiring energy, even in this disrupted cell. Surface pores have been reported to be important for nutrition and osmoregulation in other protozoa (Bokhari et al. 2008). The possibility that this viscoid extrusion represented the formation of a different morphological form, namely the development of an AF from a VF, was considered. The similar amoeboid shape recorded on a different day showed no evidence of nuclear material within it (Fig. 10) and provided no support for this hypothesis. Over the $24 \mathrm{~h}$ of the study, the material on the slide would have depleted nutrients and become progressively dehydrated, and it is possible this fluid extrusion is an osmoregulatory process occurring in a stressed VF. Blastocystis spp. have been reported to survive in varying osmotic conditions (Ho et al. 1993), although only cysts survive immersion in fresh water. Osmosensing and osmoregulation have been reported in yeasts, microalgae and protozoa (Suescun-Bolivar and Thome 2015). Protozoans have been reported to osmoregulate variously by changing the concentration of alanine, effluxing ions and water from either contractile vacuoles or intracellular compartments as well as inhibiting mitochondrial function during hyperosmolar conditions (Suescun-Bolivar and Thome 2015).

\section{Summary}

Deconvolutional microscopy of xenic cultures of Blastocystis spp. produced images of living organisms in their natural microbial environment but proved challenging due to the fragility of the organisms. Fluorescent spectrometry revealed that Blastocystis cells exhibit green autofluorescence in common with many other microalgae. GAF was able to be distinguished from fluorescein-specific Blastocystis antibody labelling in VF and GF but not cysts. Care will need to be taken in future studies to distinguish GAF from other stains fluorescing in the green light spectrum. Surface pores were observed and 
appeared to be opening and closing actively. Extrusion of a viscoid material from VF may be occurring via these pores. Coincident with the extrusion, the VF appeared to deflate, suggesting a direct connection from the surface to the central vacuole. This extrusion may be an osmoregulatory mechanism used by Blastocystis spp. to maintain cell homeostasis and integrity. Further studies could use time-lapse images to examine changes in organisms immersed in hypo-osmolar and hyperosmolar medium to further explore this hypothesis. The nature of the tear-shaped granules observed exiting from an amoebic form is unclear. Amoebic forms are common in diarrheal faeces and rarely observed in culture. It is possible these are reproductive granules occurring only in amoebic forms that have been primed by the gut faecal microbiota. Fluorescent nuclear stains to prove transmission of genetic material could be combined in future time-lapse studies of amoebic forms in xenic cultures.

Acknowledgments I am grateful to Dr. Rick Gould (Antibodies Inc, USA) for generously supplying the Blastofluor ${ }^{\circledR}$ antibody, to Dr. Lyn Knott (School of Veterinary Science, Gatton, University of Queensland) for invaluable help in setting up the microscope and to Associate Professor Kevin Tan (Department of Microbiology, National University Singapore) for helpful guidance in interpretation of some videos. These experiments comply with the current laws of the country in which they were performed.

Open Access This article is distributed under the terms of the Creative Commons Attribution 4.0 International License (http:// creativecommons.org/licenses/by/4.0/), which permits unrestricted use, distribution, and reproduction in any medium, provided you give appropriate credit to the original author(s) and the source, provide a link to the Creative Commons license, and indicate if changes were made.

\section{References}

Bokhari AA, Solomon T, Desai SA (2008) Two distinct mechanisms of transport through the plasmodial surface anion channel. J Membr Biol 226(1-3):27-34. doi:10.1007/s00232-008-9136-2

Cassidy MF, Stenzel DJ, Boreham PF (1994) Electron microscopy of surface structures of Blastocystis sp. from different hosts. Parasitol Res 80(6):505-511

Denoeud F et al (2011) Genome sequence of the stramenopile Blastocystis, a human anaerobic parasite. Genome Biol 12(3):R29. doi:10.1186/gb-2011-12-3-r29

Dogruman-Al F et al (2010) Comparison of methods for detection of Blastocystis infection in routinely submitted stool samples, and also in IBS/IBD Patients in Ankara, Turkey. PLoS ONE 5(11):e15484. doi:10.1371/journal.pone.0015484

Dunn LA, Boreham PFL, Stenzel DJ (1989) Ultrastructural variation of Blastocystis hominis stocks in culture. Int J Parasitol 19:43-56
Eroglu F, Koltas IS (2010) Evaluation of the transmission mode of B. hominis by using PCR method. Parasitol Res 107(4):841-845. doi: 10.1007/s00436-010-1937-4

Gould R, Boorom K (2013) Blastocystis surface antigen is stable in chemically preserved stool samples for at least 1 year. Parasitol Res 112:2469-2471. doi:10.1007/s00436-013-3411-6

Ho LC, Singh M, Suresh G, Ng GC, Yap EH (1993) Axenic culture of Blastocystis hominis in Iscove's modified Dulbecco's medium. Parasitol Res 79(7):614-616

Jones DR (1946) A medium for investigating the breakdown of pectin by bacteria. Nature 158(4018):625

Matsumoto Y, Yamada M, Yoshida Y (1987) Light-microscopical appearance and ultrastructure of Blastocystis hominis an intestinal parasite of man. Zentralbl Bakteriol Mikrobiol Hyg A 264:379-385

Moe KT et al (1997) Experimental Blastocystis hominis infection in laboratory mice. Parasitol Res 83(4):319-325

Olivo-Diaz A et al (2012) Findings related to IL-8 and IL-10 gene polymorphisms in a Mexican patient population with irritable bowel syndrome infected with Blastocystis. Parasitol Res 111:487-491. doi:10.1007/s00436-012-2830-0

Scanlan PD (2012) Blastocystis: past pitfalls and future perspectives. Trends Parasitol 28(8):327-334. doi:10.1016/j.pt.2012.05.001

Stensvold CR (2012) Thinking Blastocystis out of the box. Trends Parasitol 28(8):305. doi:10.1016/j.pt.2012.05.004

Stenzel DJ, Boreham PF (1996) Blastocystis hominis revisited. Clin Microbiol Rev 9(4):563-584

Suescun-Bolivar LP, Thome PE (2015) Osmosensing and osmoregulation in unicellular eukaryotes. World J Microbiol Biotechnol 31(3): 435-443. doi:10.1007/s11274-015-1811-8

Tan KS (2008) New insights on classification, identification, and clinical relevance of Blastocystis spp. Clin Microbiol Rev 21(4):639-665. doi:10.1128/CMR.00022-08, 21/4/639 [pii]

Tan KS, Stenzel DJ (2003) Multiple reproductive processes in Blastocystis: proceed with caution. Trends Parasitol 19(7):290 291, author reply 291-2 doi:S1471492203001193 [pii]

Tan TC, Suresh KG (2006) Predominance of amoeboid forms of Blastocystis hominis in isolates from symptomatic patients. Parasitol Res 98(3):189-193. doi:10.1007/s00436-005-0033-7

Tan HK, Zierdt CH (1973) Ultrastructure of Blastocystis hominis. Parasitol Res 42:315-324

Tan HK, Harrison M, Zierdt CH (1974) Freeze-etch studies of the granular and vacuolated forms of Blastocystis hominis. Parasitol Res 44: 267-278

Tan TC, Suresh KG, Thong KL, Smith HV (2006) PCR fingerprinting of Blastocystis isolated from symptomatic and asymptomatic human hosts. Parasitol Res 99(4):459-465

Tang YZ, Dobbs FC (2007) Green autofluorescence in dinoflagellates, diatoms, and other microalgae and its implications for vital staining and morphological studies. Appl Environ Microbiol 73(7):23062313. doi:10.1128/AEM.01741-06

Wang W, Owen H, Traub RJ, Cuttell L, Ipankaew T, Beilefeldt-Ohmann $\mathrm{H}$ (2014) Molecular epdiemiology of Blastocystis in pigs and their in-contact humans in Southeast Queensland, Australia, and Cambodia. Vet Parasitol. doi:10.1016/j.vetpar.2014.04.006

Yoshikawa H, Kuwayama N, Enose Y (1995a) Histochemical detection of carbohydrates of Blastocystis hominis. J Eukaryot Microbiol 42: $70-74$

Yoshikawa H, Satoh J, Enose Y (1995b) Light and electron localisation of lipids in Blastocystis hominis. J Electron Microsc 44:100-103

Zierdt CH (1991) Blastocystis hominis-past and future. Clin Microbiol Rev 4(1):61-79 